\title{
Calidad de los registros clínicos de enfermería en una institución de salud del puerto de Veracruz
}

\section{Quality of nursing clinical records in a health institution in the port of Veracruz}

DOI: $10.46932 /$ sfjdv3n1-072

Received in: Jan 30st, 2021

Accepted in: Feb 1th, 2022

\section{Román Vázquez Ovando}

Licenciado en Enfermería

Enfermero General del Hospital de Alta Especialidad de Veracruz (HAEV)

Av. 20 de Noviembre, No. 1074, Col. Centro, C.P. 91700

E-mail: rom.55vazquez@gmail.com

Jorge Arturo Alvarado Martínez

Licenciado en Enfermería y Maestro en Ciencias de Enfermería

Jefe de Enfermería del Hospital de Alta Especialidad de Veracruz (HAEV)

Av. 20 de Noviembre, No. 1074, Col. Centro, C.P. 91700

E-mail: enfveralvarado@yahoo.com.mx

\section{Graciela López Orozco}

Licenciada en Enfermería, Maestra en Ciencias de Enfermería y Doctora en Educación

Docente de tiempo completo de la Facultad de Enfermería de la Universidad Veracruzana en la Región Veracruz

Juan Enríquez s/n esquina 20 de Noviembre, Col Centro, C.P. 91700

E-mail: gralopez@uv.mx

\section{Reyna Sánchez Vázquez}

Licenciada en Enfermería, Maestra en Ciencias de la Enfermería y Doctora en Educación Subjefa de Enfermería del turno nocturno del Hospital de Alta Especialidad de Veracruz (HAEV)

Av. 20 de Noviembre, No. 1074, Col. Centro, C.P. 91700

E-mail: reyna-sanchez@live.com.mx

\section{María Angélica Ibarra Estela}

Licenciada en Enfermería y Maestra en Administración de los Servicios de Salud Docente de la Facultad de Enfermería de la Universidad Veracruzana en la Región Veracruz Juan Enríquez s/n esquina 20 de Noviembre, Col Centro, C.P. 91700

E-mail: mibarra@uv.mx

\section{Guadalupe De la Rosa Lavariega}

Licenciada en Enfermería y Maestra en Administración de Instituciones de Salud

Subjefa de Enfermería del Hospital de Alta Especialidad de Veracruz (HAEV)

Av. 20 de Noviembre, No. 1074, Col. Centro, C.P. 91700

E-mail: gdelarosa8082@gmail.com

\section{Cristhián Raúl Ochoa Alarcón}

Licenciado en Enfermería

Enfermero General del Hospital de Alta Especialidad de Veracruz (HAEV) 
Av. 20 de Noviembre, No. 1074, Col. Centro, C.P. 91700

E-mail: xtian_8a79@hotmail.com

Gloria López Mora

Licenciada en Enfermería, Maestra en Ciencias de Enfermería y Doctora en Gobierno y Administración Pública

Docente de tiempo completo de la Facultad de Enfermería de la Universidad Veracruzana en la Región Veracruz

Juan Enríquez s/n esquina 20 de Noviembre, Col Centro, C.P. 91700

E-mail: glmora@uv.mx

\begin{abstract}
RESUMEN
Introducción: El proceso enfermero es la metodología que usa enfermería para estandarizar sus cuidados y se ha establecido como la herramienta de trabajo de la profesión dentro de la hoja institucionalizada de enfermería donde se desarrollan los registros clínicos. Objetivo: Determinar la calidad de los registros clínicos de enfermería del servicio de pediatría de un hospital del puerto de Veracruz. Metodología: Se realizó un estudio cuantitativo, descriptivo de corte transversal. Resultados: Al 26.9\% de los registros clínicos no se les realizo el apartado de "Evaluación de Riesgo de Úlceras por Presión" y el "Riesgo de caídas". Conclusión: Se determinó que $11.5 \%$ de los registros clínicos eran de calidad aceptable y que el $88.5 \%$ eran de calidad buena.
\end{abstract}

Palabras clave: Registros de Enfermería, Proceso de Enfermería, Calidad de la Atención de Salud.

\begin{abstract}
Introduction: The nursing process is the methodology used by nursing to standardize its care and has been established as the profession's working tool within the institutionalized nursing sheet where clinical records are developed. Objective: To determine the quality of the nursing clinical records of the pediatric service of a hospital in the port of Veracruz. Methodology: A quantitative, descriptive, cross-sectional study was carried out. Results: $26.9 \%$ of the clinical records did not include the "Pressure Ulcer Risk Assessment" and "Risk of falls" sections. Conclusion: It was determined that $11.5 \%$ of the clinical records were of acceptable quality and $88.5 \%$ were of good quality.
\end{abstract}

Keywords: Nursing Records, Nursing Process, Quality of Health Care.

\title{
1 INTRODUCCIÓN
}

La hoja de enfermería es la herramienta laboral de gestión del cuidado institucionalizada para los enfermeros, en ella se encuentra plasmada la herramienta teórico-metodológica para el cuidado de enfermería, es decir, el proceso enfermero (Reyes, 2015). En la hoja de enfermería se encuentran los elementos del Proceso de Atención de Enfermería (PAE) y el Plan de Cuidados de Enfermería (PLACE) qué los profesionales necesitan para gestionar el cuidado de sus pacientes, a decir, la valoración, planeación, ejecución y evaluación de las acciones independientes de enfermería.

Los registros clínicos de enfermería constituyen la evidencia escrita del cuidado brindado a un paciente, éste es un excelente medio de comunicación y coordinación entre los profesionales médicos y 
la correcta implementación de la continuidad de cuidados y la seguridad del paciente (Torres, 2011). La calidad del servicio de salud se concibe como una medida del resultado del proceso y de la satisfacción de los pacientes y sus familias. (Forrellat, 2014).

\subsection{REGISTROS CLÍNICOS DE ENFERMERÍA}

En algunos países se ha determinado que el cumplimiento de los registros clínicos no garantiza que estos sean de buena calidad, debido a que en ocasiones, a pesar del alto porcentaje de cumplimiento existe deficiencia en la justificación de las acciones de enfermería, indicaciones médicas y la presencia de marcas de borrado en los registros, por lo anterior, investigadores sugieren la implementación de acciones encaminadas a realizar el registro de enfermería con excelencia (Almeida et al, 2019).

Algunos estudios sobre los registros clínicos de enfermería han demostrado que hay poca coherencia de las intervenciones con el diagnóstico enfermero en los registros de clínicos, algunos diagnósticos son mal elaborados o están incompletos, esto refleja incompetencia del personal de enfermería para el manejo del proceso enfermero y por ende falta de razonamiento crítico en la muestra estudiada (Ruiz et al, 2017).

En algunas investigaciones se ha observado que el personal que participa en la realización de los registros clínicos tiene el nivel de conocimiento adecuado para desarrollar dichos registros, de tal forma se identifica que en la práctica muchos de ellos no llevaban a cabo el proceso correctamente, resultando en registros clínicos deficientes (Bautista et al, 2016).

Los errores del personal de salud pueden estar relacionados con la equivocada identificación del paciente, pues institucionalmente este proceso se realiza verificando membrete del paciente, y los datos de la hoja de enfermería, pero también a la hora de transcribir indicaciones escritas, quizá por la legibilidad de la letra del médico se escribe en la hoja de enfermería el medicamento equivocado, estos errores y algunos otros han resultado en la creación de programas de salud para disminuir los errores cometidos por el personal de hospitales o unidades de salud (Consejo de salubridad, 2017).

La hoja de enfermería deberá elaborarse por el personal en turno, según lo establecido por las normas internas del establecimiento y órdenes del médico, esta deberá contener como mínimo habitus exterior, gráfica de signos vitales, ministración de medicamentos, fecha, hora, cantidad, vía prescrita, procedimientos realizados y observaciones, deberán expresarse en lenguaje técnico-médico, sin abreviaturas, con letra legible, sin enmendaduras ni tachaduras y conservarse en buen estado (Norma oficial mexicana NOM-004-ssa3-2012, de los servicios de planificación familiar). 


\subsection{MODELO DEL CUIDADO DE ENFERMERÍA}

El modelo del cuidado de enfermería tiene enfoque integral y sistémico aplicable a los tres niveles de atención en salud fortaleciendo con ello la toma de decisiones en atención primaria a la salud, éste modelo del cuidado de enfermería se integra por cuatro elementos y similares básicos de la enfermería aplicables en el ámbito ambulatorio y hospitalario, estos son: el Metaparadigma de enfermería o supuestos principales, Proceso Atención de Enfermería (PAE), Plan de Cuidados de Enfermería (PLACE) y las recomendaciones de las Guías de Práctica Clínica de Enfermería (GPCE) (Secretaría de salud, 2018).

El modelo de atención de enfermería integra intervenciones basadas en la evidencia científica, utiliza y adopta el contenido de las clasificaciones de la Asociación Norteamericana de Diagnósticos de Enfermería (NANDA), Resultados de Enfermería (NOC), Clasificación de Intervención de Enfermería (NIC), ya que las tres clasificaciones contribuyen a la homologación del lenguaje y estandarización del cuidado (Secretaría de salud, 2018).

En países de Latino América han aceptado que el uso del proceso de enfermería favorece el desarrollo de los sistemas de clasificación para la documentación de los registros clínicos de enfermería. Por ello el uso de la Clasificación Internacional para la Práctica de Enfermería (CIPE) puede colaborar en la autonomía de la enfermería para planificar actividades en el cuidado de la mujer embarazada usando un lenguaje específico (Caniçali et al, 2015).

El proceso enfermero es esencial para gestionar el cuidado de los pacientes, así mismo el profesional de enfermería puede sistematizar acciones que permiten coadyuvar al bienestar de los pacientes, esto ha quedado demostrado en la actual pandemia de COVID 19, pues gracias a los registros clínicos de enfermería, se podrán obtener hallazgos clínicos que permitirán generar datos epidemiológicos (Reis et al, 2020).

La existencia de la NOM del Expediente Clínico, a pesar de ser difundida y normatizada en las Instituciones de salud, su uso y manejo cotidiano no es el adecuado, parece ser que existe un vacío en la normativa, asimismo esto incluye a los comités encargados de la calidad en este rubro. El nivel de cumplimiento de los expedientes clínicos se encuentra en una categoría de regular (Carmona, \& Ponce, 2014).

\section{METODOLOGÍA}

\subsection{TIPO Y DISEÑO DE ESTUDIO}

La presente investigación es cuantitativa, descriptiva de corte transversal (Hernández et al, 2014).). Es cuantitativa porque se definieron las variables y se les otorgó un valor que fue analizado 
estadísticamente. Descriptiva porque los resultados fueron interpretados y de corte transversal por que el estudio se realizó en un momento específico.

\subsection{UNIVERSO}

El universo objeto de investigación fueron las hojas de enfermería con los registros clínicos del servicio de pediatría de un hospital del Puerto de Veracruz, México.

\subsection{MUESTREO}

El muestreo que se utilizó fue no probabilístico a conveniencia del investigador porque la muestra se conformó del 100\% de la población estudiada.

\subsection{MUESTRA}

La muestra quedó conformada por 25 hojas de enfermería con los registros clínicos del servicio de pediatría de un hospital del puerto de Veracruz, México.

\subsection{CRITERIOS DE SELECCIÓN}

Criterios de inclusión: Registros clínicos del servicio de pediatría.

Criterios de exclusión: Registros clínicos que no hayan estado disponibles para su evaluación.

Criterios de eliminación: Registros clínicos por motivo de que el investigador haya dejado incompleta la cédula de evaluación.

\subsection{INSTRUMENTO}

Para la presente investigación se utilizó una cédula de evaluación titulada "Instrumento de Evaluación de Registros Clínicos de Enfermería”, la cual tiene una sola dimensión y 22 Ítems que evalúan la calidad de los registros clínicos de enfermería. Cada uno de los ítems tiene la opción de seleccionar una respuesta para lo cual se dan cuatro opciones de tipo Likert, dichas opciones de respuestas son: 1: "No realizado", 2: "Mala", 3: Aceptable, 4: Buena. Lo anterior se interpreta que a mayor puntaje la calidad de los registros clínicos es mejor.

Para analizar la fiabilidad del instrumento se utilizó el programa estadístico "statistical package for social sciences" (SPSS) y se obtuvo un alfa de chronbach de 0.71, el cual fue aceptable para realizar la presente investigación. 


\subsection{PROCEDIMIENTO Y ANÁLISIS ESTADÍSTICO}

El presente trabajo de investigación surge por la necesidad de saber si el personal de enfermería realiza registros clínicos de manera eficiente, para ello se analizaron los productos del trabajo de los enfermeros, es decir, los registros clínicos de enfermería. Se estructuró el planteamiento del problema, seguidamente se creó la pregunta de investigación, posterior a ello se establecieron los objetivos de éste proyecto, luego se redactaron las hipótesis de investigación y más tarde se establecieron las variables dependiente e independiente.

Se definió el tipo de estudio y el diseño que tendría, también se delimitó la población objeto de estudio y se formularon los criterios de selección. Se seleccionó el instrumento para la recopilación de datos y fue aplicado por los investigadores a los registros clínicos que formaron parte de la muestra.

Para el análisis de datos se utilizó el programa estadístico "statistical package for social sciences" con el cual se analizó la fiabilidad del instrumento y se usó estadística descriptiva para analizar los resultados obtenidos en la recogida de datos, para finalmente interpretar los resultados y discutir las conclusiones de la investigación, así como definir si se cumplieron los objetivos, las hipótesis y responder la pregunta de investigación.

\subsection{CONSIDERACIONES ÉTICAS}

Para la presente investigación se consideraron aspectos importantes de la Constitución Política de los Estados Unidos Mexicanos en el Artículo $4^{\circ}$ (Constitución Política de los Estados Unidos Mexicanos, 2021), el Reglamento de la Ley General de Salud en Materia de Investigaciones para la Salud (Reglamento de la Ley General de Salud en Materia de Investigaciones para la Salud, 2014), el Código Deontológico de Enfermería (Consejo Internacional de Enfermeras, 2017). el Código de Helsinki (Comisión Nacional de Arbitraje Médico, 2013) y la NORMA Oficial Mexicana NOM-012-SSA3-2012, que establece los criterios para la ejecución de proyectos de investigación para la salud en seres humanos (NORMA Oficial Mexicana NOM-012-SSA3-2012, que establece los criterios para la ejecución de proyectos de investigación para la salud en seres humanos).

\section{RESULTADOS}

Al 3.8\% de los registros clínicos no se le desarrolló el apartado de la hoja de enfermería donde van los “Datos de Identificación del paciente, Unidad, Fecha y Días de Hospitalización” mientras que el 11.5\% de los registros clínicos en este apartado fue clasificado con mala calidad, el $61.5 \%$ con calidad aceptable y el $23.1 \%$ con buena calidad. Al $7.7 \%$ de los registros clínicos no se le desarrolló este apartado los 
"Signos Vitales" y el 15.4\% de los registros clínicos en este apartado fueron clasificados con mala calidad, mientras que el $38.5 \%$ fueron clasificados como aceptables y otro $38.5 \%$ con buena calidad.

Al 23.1\% de los registros clínicos (n=26) no se le desarrolló el apartado de la Escala Análoga del Dolor" mientras que el $15.4 \%$ de los registros clínicos en este apartado fueron clasificados con mala calidad, el 34.6\% como aceptables y el $26.9 \%$ con buena calidad. Los resultados obtenidos muestran que en el apartado de "Somatometría" al 7.7\% de los registros clínicos no se le desarrolló este apartado de la hoja de enfermería mientras que el $11.5 \%$ de los registros clínicos en este apartado fueron clasificados con mala calidad, el $65.4 \%$ como aceptables y el $15.4 \%$ con buena calidad.

Al 15.4\% de los registros clínicos no se le desarrolló el aparato de "Ingresos Enterales y Parenterales". Un $15.4 \%$ de los registros clínicos en este apartado fueron clasificados con mala calidad, mientras que el 50.0\% fueron clasificados como aceptables y el 19.2\% con buena calidad. El 15.4\% de los registros clínicos no tenían desarrollado el apartado de "Egresos, el 15.4\% de los registros clínicos en este apartado fueron clasificados con mala calidad, mientras que el $42.3 \%$ como aceptables y el $26.9 \%$ con buena calidad.

El $15.4 \%$ de los registros clínicos no tenía desarrollado el apartado de "Laboratorios, Pruebas Biológicas, Reactivos, Estudios y Operaciones”, el 7.7\% de los registros clínicos en este apartado fueron clasificados con mala calidad, en tanto que el $23.1 \%$ como aceptables y el $26.9 \%$ con buena calidad. El 15.4\% de los registros clínicos no tenía desarrollado el apartado de "Esquema Terapéutico”, el 11.5\% de los registros en este apartado fueron clasificados con mala calidad, mientras que el $38.5 \%$ como aceptables y el $34.6 \%$ con buena calidad.

Los resultados obtenidos muestran que en el apartado "Patrones Funcionales" el $19.2 \%$ de los registros clínicos no estaban desarrollados, mientras que el $11.5 \%$ de los registros en este apartado fueron clasificados con mala calidad, el 53.8\% como aceptables y el $15.4 \%$ con buena calidad. En $15.4 \%$ de los registros clínicos no se desarrolló el apartado "Signos y Síntomas”, el 34.6\% de los registros clínicos en este apartado fueron clasificados con aceptable calidad, y el 50\% con buena calidad.

Al 15.4\% de los registros clínicos no se le desarrollo el apartado de "Diagnóstico de Enfermería" mientras que el $50 \%$ de los registros clínicos en este apartado fueron clasificados con aceptable calidad y el 34.6\% con buena calidad. Al 15.4\% de los registros clínicos no se le desarrollo el apartado "Intervención de Colaboración", mientras el $11.5 \%$ de los registros clínicos en este apartado fueron clasificados con mala calidad, el $30.8 \%$ como aceptables y el $42.3 \%$ con buena calidad.

Al 11.5\% de los registros clínicos no se le desarrolló el apartado "Respuestas y Evolución", mientras que el $3.8 \%$ de los registros clínicos en este apartado fueron clasificados con mala calidad, el 46.2\% como aceptables y el 38.5\% con buena calidad. Al 3.8\% de los registros clínicos no se le desarrolló 
el apartado "Observaciones", mientras que el $42.3 \%$ de los registros clínicos en este apartado fueron clasificados con aceptable calidad y el $53.8 \%$ con buena calidad.

Al 7.7\% de los registros clínicos no se le desarrolló el apartado "Plan de Alta", mientras que el $57.7 \%$ de los registros clínicos en este apartado fueron clasificados con aceptable calidad, y el 34.6\% con buena calidad. Al 3.8\% de los registros clínicos no se le desarrolló el apartado "Inicial del Nombre, Apellido y Matrícula", mientras que el 38.5\% de los registros clínicos en este apartado fueron clasificados con aceptable calidad, y el $57.7 \%$ con buena calidad. El $34.6 \%$ de los registros clínicos en el apartado "Pulcritud y Legibilidad" fueron clasificados con aceptable calidad, y el $65.4 \%$ fueron clasificados con buena calidad.

\section{DISCUSIÓN}

En un estudio similar al presente trabajo de investigación que fue realizado por López et al. (2015), los autores concluyeron que el personal de enfermería no realiza registros clínicos de calidad pues los resultados arrojaron qué en la clasificación obtenida del estudio, el 65.84\% de la calidad de los registros clínicos se estableció en "No cumplimiento", lo cual deja al descubierto que en su mayoría el personal no cumple con los requisitos del proceso.

Caso contrario a este estudio donde el nivel de calidad de los registros clínicos fue bueno en un $88.5 \%$ y aceptable en un $11.5 \%$. Esto demuestra que el personal de enfermería está realizando registros clínicos de calidad y que está cumpliendo con el proceso y sus requisitos mínimos para gestionar el cuidado de sus pacientes y así procurar el bienestar y el restablecimiento de la salud de ellos.

\section{CONCLUSIÓN}

La calidad de los registros clínicos de enfermería fue "Buena" en el $88.5 \%$ de los registros analizados, y el $11.5 \%$ de estos obtuvo un nivel aceptable. Por lo anterior se concluye que el personal de enfermería del servicio de pediatría del hospital donde se hizo el estudio está realizando registros clínicos de buena calidad. Esto es de amplio beneficio para los pacientes, pues de esta manera el personal está optimizando los procesos de gestión del cuidado de sus pacientes.

Las debilidades de enfermería en la realización son mínimos, pero importantes de pulir, ya que el $19.2 \%$ de los registros no tenía realizado intervenciones para el control del dolor ni las actividades de enfermería y el $26.9 \%$ no tenía realizada el apartado de los problemas interdependientes. Es evidente que los pocos errores de enfermería en la realización de los registros clínicos tienen que ver con el "No hacer" y habría que determinar esta situación con otra investigación para mantener y mejorar el nivel de calidad de los registros clínicos de enfermería. 


\section{Calidad de los Registros Clínicos de} Enfermería

\section{$11 \%$}

Aceptable

$89 \%$ 


\section{REFERENCIAS}

1. Almeida, V., Santos, R., Silva, L., De Jesús, N., Marques, C., \& Da silva, L. G. (2019). Auditoria da qualidade dos registros de enfermagem em prontuários em um hospital universitário. Enfermagem em Foco. 10 (3): 28-33.

2. Bautista, L. M., Vejar, L.Y., Pabón, M. R., Moreno, J. J., Fuentes, L., León, K. Y., \& Bonilla, J. A. (2016). Grado de adherencia al protocolo de registros clínicos de enfermería. Revista CUIDARTE. 7(1): 1195-203. http://dx.doi.org/10.15649/cuidarte.v7i1.237.

3. Caniçali, C., Cassaro, C., Cândida, J., Marabotti, F., De Sousa, M. V., \& Almeida, E. F. (2015). Classificação internacional para a prática de enfermagem na. Enfermagem em Foco. 6(1/4): 17-23.

4. Carmona, B., \& Ponce, G. (2014). Evaluación del expediente clínico integrado y de calidad en pacientes de cirugía programada. Revista CONAMED. 19(3): 117-127.

5. Comisión Nacional de Arbitraje Médico. (2013). Declaración de Helsinki de la Asociación Médica Mundial. Recomendaciones para guiar a los médicos en la investigación biomédica en personas. http://www.conamed.gob.mx/prof_salud/pdf/helsinki.pdf

6. Consejo de Salubridad General [CSG] (2017). Acciones esenciales para la seguridad del paciente. $\begin{array}{lllll}\text { Diario Oficial la } & \text { de } & \text { Federación }\end{array}$ https://www.dof.gob.mx/nota_detalle.php?codigo=5496728\&fecha=08/09/2017.

7. Consejo Internacional de Enfermeras. (2017). Código deontológico del Consejo Internacional de Enfermeras para la profesión de enfermería. RevEnferm Inst Mex Seguro Soc. 25(2):83-4. https://www.medigraphic.com/pdfs/enfermeriaimss/eim-2017/eim172b.pdf

8. Forrellat, M. (2014). Calidad en los servicios de salud: un reto ineludible. Revista Cubana Hematología, Inmunología y Hemoterapia. Vol. 30(2):179-183

9. Hernández, R., Fernández, C. \& Baptista, P. (2014). Metodología de la investigación. McGRAWHILL. ISBN: 978-1-4562-2396-0.

10. Norma oficial mexicana NOM-004-ssa3-2012, de los servicios de planificación familiar. Modificada, Diario Oficial de la Federación [D.O.F.], 15 de octubre de 2012 (México). http://dof.gob.mx/nota_detalle.php?codigo=5272787\&fecha=15/10/2012.

11. NORMA Oficial Mexicana NOM-012-SSA3-2012, que establece los criterios para la ejecución de proyectos de investigación para la salud en seres humanos. Diario Oficial de la Federación [D.O.F.], 04 de enero de 2013 (México)

12. Reglamento de la Ley General de Salud en Materia de Investigaciones para la Salud [R.L.G.S.M.I.S.], Reformada, Diario Oficial de la Federación [D.O.F.], 7 de febrero de 1984 (México).

13. Reis, A., Alves, G. L. Da silva, R. S., \& De Santana, E. S. (2020). Reflexões sobre o processo de enfermagem no trabalho de enfermeiras frente à pandemia da covid-19. Enfermagem em Foco. 11(1): 6267.

14. Reyes, E. (2015). Fundamentos de Enfermería. Ciencia, Metodologia y Tecnologia (2da ed.). Manual Moderno. 
15. Ruiz, L. A., Gómez, J. M., Cuevas, L., \& Martínez, A. L. (2017). Coherencia diagnostico/intervención de enfermería en la hoja de registros clínicos y uso de lenguaje estandarizado. Revista CONAMED. 22(4), $161-165$.

16. Secretaría de Salud. (2018). Modelo del cuidado de enfermería. Programa Nacional de Enfermería. Obtenido de http://www.cpe.salud.gob.mx/site3/programa/modelo_cuidado_enfermeria.html.

17. Secretaría de Salud. (2018). Modelo del cuidado de enfermería. Subsecretaría de integración y desarrollo.

Primera edición.

Obtenido

de http://www.calidad.salud.gob.mx/site/editorial/docs/modelo_cuidado_enfermeria.pdf

18. Torres, M., Zarate, R. A., \& Matus, R. (2011). Calidad de los registros clínicos de enfermería: Elaboración de un instrumento para su evaluación. Enfermería Universitaria. 8(1): 17-25. 